\title{
Spherical structures on torus knots and links *
}

\author{
Alexander Kolpakov, Alexander Mednykh
}

\begin{abstract}
The present paper considers two infinite families of cone-manifolds endowed with spherical metric. The singular strata is either the torus knot $\mathrm{t}(2 n+1,2)$ or the torus link $\mathrm{t}(2 n, 2)$. Domains of existence for a spherical metric are found in terms of cone angles and volume formulæ are presented.
\end{abstract}

Key words: Spherical geometry, cone-manifold, knot, link.

\section{Introduction}

A three-dimensional cone-manifold is a metric space obtained from a collection of disjoint simplices in the space of constant sectional curvature $k$ by isometric identification of their faces in such a combinatorial fashion that the resulting topological space is a manifold (also called the underlying space for a given cone-manifold).

Such the metric space inherits the metric of sectional curvature $k$ on the union of its 2- and 3-dimensional cells. In case $k=+1$ the corresponding cone-manifold is called spherical (or admits a spherical structure). By analogy, one defines euclidean $(k=0)$ and hyperbolic $(k=-1)$ cone-manifolds.

The metric structure around each 1-cell is determined by a cone angle that is the sum of dihedral angles of corresponding simplices sharing the 1-cell under identification. The singular locus of a cone-manifold is the closure of all its 1-cells with cone angle different from $2 \pi$. For the further account we suppose that every component of the singular locus is an embedded circle with constant cone angle along it.

A particular case of cone-manifold is an orbifold with cone angles $2 \pi / m$, where $m$ is an integer (cf. [1]).

The present paper considers two infinite families of cone-manifolds with underlying space the three-dimensional sphere $\mathbb{S}^{3}$. The first family consists of cone-manifolds with singular locus the torus knot $\mathrm{t}(2 n+1,2)$ with $n \geq 1$. In the rational census 2 these knots are denoted by $(2 n+1) / 1$. The second family of cone-manifolds consists of those with singular locus a two-component torus link $\mathrm{t}(2 n, 2)$ with $n \geq 2$. These links are two-bridge and correspond to the links

${ }^{*}$ The work is performed under auspices of the Swiss National Science Foundation no. 200020-113199/1, "Scientific Schools"-5682.2008.1 and RFBR no. 06-01-00153. 
$2 n / 1$ in the rational census. The simplest examples of such the knots and links are the trefoil knot 3/1 and the link 4/1. In the Rolfsen table [2] one finds them as the knot $3_{1}$ and the link $4_{1}^{2}$.

By the Theorem of $\mathrm{W}$. Thurston 3 , the manifold $\mathbb{S}^{3} \backslash 3_{1}$ does not admit a hyperbolic structure. However, it admits two other geometric structures [4]: $\mathbb{H}^{2} \times \mathbb{R}$ and $\widetilde{\mathrm{PSL}}(2, \mathbb{R})$. It follows from the paper $[5$ that the spherical dodecahedron space (i.e. Poincaré homology sphere) is a cyclic 5 -fold covering of $\mathbb{S}^{3}$ branched over $3_{1}$. Thus, the orbifold $3_{1}\left(\frac{2 \pi}{5}\right)$ with singular locus the trefoil knot and cone angle $\frac{2 \pi}{5}$ is spherical. Due to the Dunbar's census [6], orbifold $3_{1}\left(\frac{2 \pi}{n}\right)$ is spherical if $n \leq 5$, Nil-orbifold if $n=6$ and $\widetilde{\mathrm{PSL}}(2, \mathbb{R})$-orbifold if $n \geq 7$. Spherical structure on the cone-manifold $3_{1}(\alpha)$ with underlying space the three-dimensional sphere $\mathbb{S}^{3}$ is studied in [7].

The consideration of two-bridge torus links is carried out starting with the simplest one possessing non-abelian fundamental group, namely $4_{1}^{2}$.

The previous investigation on spherical structures for cone-manifolds is carried out mainly in the papers $[8,9,10$. The present paper develops a method to analyse existence of a spherical metric for two-bridge torus knot and link conemanifolds. Also, the lengths of singular geodesics are calculated and the volume formulæ are obtained (cf. Theorem 1 and Theorem 2).

\section{$2 \quad$ Projective model $\mathbb{S}_{\lambda}^{3}$}

The purpose of the present section is to construct the projective model $\mathbb{S}_{\lambda}^{3}$ that one can use to study geometric properties of two-bridge torus knots and links and to build up holonomy representation for the corresponding cone-manifolds. Other projective models for homogeneous geometries are described in 11. Consider the set $\mathbb{C}^{2}=\left\{\left(z_{1}, z_{2}\right): z_{1}, z_{2} \in \mathbb{C}\right\}$ as a four-dimensional vector space over $\mathbb{R}$. We denote it by $\mathbb{C}_{\mathbb{R}}^{2}$ and equip with Hermitian product

$$
\left\langle\left(z_{1}, z_{2}\right),\left(w_{1}, w_{2}\right)\right\rangle_{\mathrm{H}}=\left(z_{1}, z_{2}\right) \mathcal{H}{\overline{\left(w_{1}, w_{2}\right)}}^{T}
$$

where

$$
\mathcal{H}=\left(\begin{array}{cc}
1 & \lambda \\
\lambda & 1
\end{array}\right)
$$

is a symmetric matrix with $-1<\lambda<+1$.

The natural inner product is associated to the Hermitian form above:

$$
\left\langle\left(z_{1}, z_{2}\right),\left(w_{1}, w_{2}\right)\right\rangle=\operatorname{Re}\left\langle\left(z_{1}, z_{2}\right),\left(w_{1}, w_{2}\right)\right\rangle_{\mathrm{H}}
$$

and the respective norm is

$$
\left\|\left(z_{1}, z_{2}\right)\right\|=\left|z_{1}\right|^{2}+\left|z_{2}\right|^{2}+\lambda\left(z_{1} \bar{z}_{2}+\bar{z}_{1} z_{2}\right)
$$

Call two elements $\left(z_{1}, z_{2}\right)$ and $\left(w_{1}, w_{2}\right)$ in $\stackrel{\circ}{\mathbb{C}_{\mathbb{R}}^{2}}=\mathbb{C}_{\mathbb{R}}^{2} \backslash(0,0)$ equivalent if there is $\mu>0$ such that $\left(z_{1}, z_{2}\right)=\left(\mu w_{1}, \mu w_{2}\right)$. We denote this equivalence relation as $\left(z_{1}, z_{2}\right) \sim\left(w_{1}, w_{2}\right)$. 
Identify the factor-space $\stackrel{\circ}{\mathbb{C}_{\mathbb{R}}^{2}} / \sim$ with the three-dimensional sphere

$$
\mathbb{S}_{\lambda}^{3}=\left\{\left(z_{1}, z_{2}\right) \in \mathbb{C}_{\mathbb{R}}^{2}:\left\|\left(z_{1}, z_{2}\right)\right\|=1\right\},
$$

endowed with the Riemannian metric

$$
\mathrm{d} s_{\lambda}^{2}=\left|\mathrm{dz}_{1}\right|^{2}+\left|\mathrm{dz_{2 }}\right|^{2}+\lambda\left(\mathrm{dz}_{1} \mathrm{~d} \bar{z}_{2}+\mathrm{dz}_{1} \mathrm{dz}_{2}\right) .
$$

By means of equality

$$
\mathrm{d} s_{\lambda}^{2}=\frac{1+\lambda}{2}\left|\mathrm{~d} z_{1}+\mathrm{d} z_{2}\right|^{2}+\frac{1-\lambda}{2}\left|\mathrm{~d} z_{1}-\mathrm{d} z_{2}\right|^{2},
$$

the linear transformation

$$
\xi_{1}=\sqrt{\frac{1+\lambda}{2}}\left(z_{1}+z_{2}\right), \quad \xi_{2}=\sqrt{\frac{1-\lambda}{2}}\left(z_{1}-z_{2}\right)
$$

provides an isometry between $\left(\mathbb{S}_{\lambda}^{3}, \mathrm{~d} s_{\lambda}^{2}\right)$ and $\left(\mathbb{S}^{3}, \mathrm{~d} s^{2}\right)$, where $\mathrm{d} s^{2}=\left|\mathrm{d} \xi_{1}\right|^{2}+$ $\left|\mathrm{d} \xi_{2}\right|^{2}$ is the standard metric of sectional curvature +1 on the unit sphere $\mathbb{S}^{3}=$ $\left\{\left(\xi_{1}, \xi_{2}\right) \in \mathbb{C}^{2}:\left|\xi_{1}\right|^{2}+\left|\xi_{2}\right|^{2}=1\right\}$.

Let $P, Q$ be two points in $\mathbb{S}_{\lambda}^{3}$. The spherical distance between $P$ and $Q$ is a real number $d_{\lambda}(P, Q)$ that is uniquely determined by the conditions $0 \leq d_{\lambda}(P, Q) \leq$ $\pi$ and $\cos d_{\lambda}(P, Q)=\langle P, Q\rangle$.

\section{Torus knots $\mathbb{T}_{n}$}

Let $\mathbb{T}_{n}, n \geq 1$ be the torus knot $\mathrm{t}(2 n+1,2)$ embedded in $\mathbb{S}^{3}$. The knot $\mathbb{T}_{n}$ is the two-bridge knot $(2 n+1) / 1$ in the rational census (Fig. 1). Let $\mathbb{T}_{n}(\alpha)$ denote a cone-manifold with singular locus $\mathbb{T}_{n}$ and the cone angle $\alpha$ along it.

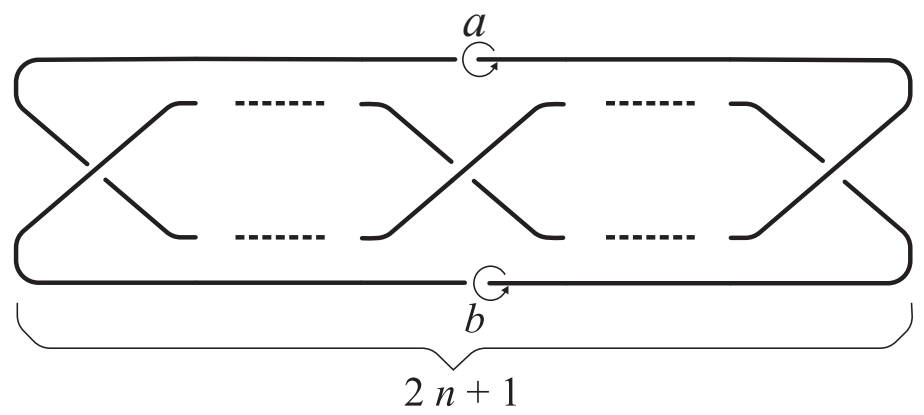

Figure 1: Knot $(2 n+1) / 1$

The aim of the present section is to investigate cone-manifolds $\mathbb{T}_{n}(\alpha), n \geq 1$ to find out the domain of sphericity in terms of the cone angle and to derive the volume formulæ.

Two lemmas precede the further exposition: 
Lemma 1 For every $0<\alpha<2 \pi$ and $-1<\lambda<+1$ the linear transformations

$$
A=\left(\begin{array}{cc}
1 & 0 \\
-2 i e^{i \frac{\alpha}{2}} \lambda \sin \frac{\alpha}{2} & e^{i \alpha}
\end{array}\right)
$$

and

$$
B=\left(\begin{array}{cc}
e^{i \alpha} & -2 i e^{i \frac{\alpha}{2}} \lambda \sin \frac{\alpha}{2} \\
0 & 1
\end{array}\right)
$$

are isometries of $\mathbb{S}_{\lambda}^{3}$.

Proof. For the further account let us assume that the multiplication of vectors by matrices is to the right. A linear transformation $L$ of the space $\mathbb{C}_{\mathbb{R}}^{2}$ preserves the corresponding Hermitian form if and only if for every pair of vectors $P, Q \in$ $\mathbb{C}_{\mathbb{R}}^{2}$ it holds that

$$
\langle P, Q\rangle_{\mathrm{H}}=P \mathcal{H} \bar{Q}^{T}=P L \mathcal{H} \bar{L}^{T} \bar{Q}^{T}=\langle P L, Q L\rangle_{\mathrm{H}} .
$$

The condition above is equivalent to

$$
\mathcal{H}=L \mathcal{H} \bar{L}^{T} .
$$

In particular,

$$
\cos d_{\lambda}(P, Q)=\langle P, Q\rangle=\langle P L, Q L\rangle=\cos d_{\lambda}(P L, Q L),
$$

that means $L$ preserves the spherical distance between $P$ and $Q$.

Let $L=A$ and $L=B$ in series, one verifies that $A$ and $B$ preserve the Hermitian norm on $\mathbb{C}_{\mathbb{R}}^{2}$ and, consequently, the spherical distance on $\mathbb{S}_{\lambda}^{3}$.

Lemma 2 Let $A$ and $B$ be the same matrices as in the affirmation of Lemma 1 . Then for all integer $n \geq 1$ one has

$$
(A B)^{n} A-B(A B)^{n}=2 U_{2 n}(\Lambda) e^{i \frac{(2 n+1)(\pi+\alpha)}{2}} \sin \frac{\alpha}{2} M,
$$

where $M$ is a non-zero $2 \times 2$-matrix and $U_{2 n}(\Lambda)$ is the second kind Chebyshev polynomial of power $2 n$ in variable $\Lambda=\lambda \sin \frac{\alpha}{2}$.

Proof. As far as $-1<\lambda<+1$, one obtains

$$
-1<\Lambda=\lambda \sin \frac{\alpha}{2}<+1 .
$$

Substitute

$$
\Lambda=\cos \theta
$$

with the unique $0<\theta<\pi$.

Then matrices $A$ and $B$ are rewritten in the form

$$
A=\left(\begin{array}{cc}
1 & 0 \\
-2 i e^{i \frac{\alpha}{2}} \cos \theta & e^{i \alpha}
\end{array}\right),
$$




$$
B=\left(\begin{array}{cc}
e^{i \alpha} & -2 i e^{i \frac{\alpha}{2}} \cos \theta \\
0 & 1
\end{array}\right) .
$$

On purpose to diagonalize the matrix $A B$, use

$$
V=\left(\begin{array}{cc}
i e^{-i \frac{\alpha}{2}} e^{-i \theta} & i e^{-i \frac{\alpha}{2}} e^{i \theta} \\
1 & 1
\end{array}\right),
$$

and obtain

$$
D=V^{-1}(A B) V=\left(\begin{array}{cc}
-e^{i \alpha} e^{2 i \theta} & 0 \\
0 & -e^{i \alpha} e^{-2 i \theta}
\end{array}\right) .
$$

Note, that $V$ might be not an isometry, but it is utile for computation.

Thus

$$
\begin{gathered}
(A B)^{n} A-B(A B)^{n}=\left(V D^{n} V^{-1}\right) A-B\left(V D^{n} V^{-1}\right)= \\
=2 \frac{\sin (2 n+1) \theta}{\sin \theta} e^{i \frac{(2 n+1)(\pi+\alpha)}{2}} \sin \frac{\alpha}{2}\left(\begin{array}{cc}
-1 & \lambda \\
-\lambda & 1
\end{array}\right)= \\
=2 U_{2 n}(\cos \theta) e^{i \frac{(2 n+1)(\pi+\alpha)}{2}} \sin \frac{\alpha}{2} M=2 U_{2 n}(\Lambda) e^{i \frac{(2 n+1)(\pi+\alpha)}{2}} \sin \frac{\alpha}{2} M,
\end{gathered}
$$

with the matrix

$$
M=\left(\begin{array}{ll}
-1 & \lambda \\
-\lambda & 1
\end{array}\right)
$$

as the present Lemma claims.

The main theorem of the section follows:

Theorem 1 The cone-manifold $\mathbb{T}_{n}(\alpha), n \geq 1$ is spherical if

$$
\frac{2 n-1}{2 n+1} \pi<\alpha<2 \pi-\frac{2 n-1}{2 n+1} \pi .
$$

The length of its singular geodesic (i.e. the length of the knot $\mathbb{T}_{n}$ ) equals

$$
l_{\alpha}=(2 n+1) \alpha-(2 n-1) \pi .
$$

The volume of $\mathbb{T}_{n}(\alpha)$ is

$$
\mathbb{V o l} \mathbb{T}_{n}(\alpha)=\frac{1}{2 n+1}\left(\frac{2 n+1}{2} \alpha-\frac{2 n-1}{2} \pi\right)^{2} .
$$

Proof. The fundamental group of the knot $\mathbb{T}_{n}$ is presented as

$$
\pi_{1}\left(\mathbb{S}^{3} \backslash \mathbb{T}_{n}\right)=\left\langle a, b \mid(a b)^{n} a=b(a b)^{n}\right\rangle,
$$

with generators $a$ and $b$ as at Fig. 1 .

Since the cone-manifold $\mathbb{T}_{n}(\alpha)$ admits a spherical structure, then there exists a holonomy mapping [1, that is a homomorphism

$$
h: \pi_{1}\left(\mathbb{S}^{3} \backslash \mathbb{T}_{n}\right) \longmapsto \operatorname{Isom} \mathbb{S}_{\lambda}^{3} .
$$


We will choose $h$ in respect with geometric construction of the cone-manifold. All the further computations to find the length of the knot $\mathbb{T}_{n}$ and the volume of the cone-manifold $\mathbb{T}_{n}(\alpha)$ are performed making use of the corresponding fundamental polyhedron $\mathcal{P}_{n}$ (Fig. 2). The construction algorithm for the polyhedron is given in [12].

The combinatorial polyhedron $\mathcal{P}_{n}$ has vertices $P_{i}, i \in\{1, \ldots, 4 n+2\}$ and edges $P_{i} P_{i+1}, i \in\{1, \ldots, 4 n+2\}$, with $P_{4 n+3}=P_{1}$, also $P_{1} P_{2 n+2}$ and $P_{2} P_{2 n+3}$. Let $N, S$ denote the middle points (the North and the South poles of $\mathcal{P}_{n}$ ) on the edges $P_{1} P_{2 n+2}$ and $P_{2} P_{2 n+3}$, respectively. Then, consider also edges $N P_{i}, S P_{i}$, $i \in\{1, \ldots, 4 n+2\}$.

Without loss in generality, choose the holonomy representation such that

$$
h(a)=A, h(b)=B,
$$

where $A$ and $B$ are matrices from Lemma 1.

The generators of the fundamental group for $\mathbb{T}_{n}$ under the holonomy mapping $h$ correspond to isometries acting on $\mathcal{P}_{n}$. These isometries identify its faces by means of rotation about the edge $P_{1} P_{2 n+2}$ for the top "cupola" of $\mathcal{P}_{n}$ and rotation about $P_{2} P_{2 n+3}$ for the bottom one (see, Fig. 2). Then the edges $P_{1} P_{2 n+2}$ and $P_{2} P_{2 n+3}$ knot itself to produce $\mathbb{T}_{n}$ (cf. [12, 13]).

In order to construct the polyhedron $\mathcal{P}_{n}$ assume that its edge $P_{1} P_{2}$ is given by

$$
P_{1}=(1,0), P_{2}=(0,1) .
$$

Then one has

$$
\cos d_{\lambda}\left(P_{1}, P_{2}\right)=\left\langle P_{1}, P_{2}\right\rangle=\lambda,
$$

i.e. the spherical distance between the points $P_{1}$ and $P_{2}$ can vary from 0 to $\pi$. Thus, prescribing certain coordinates to the end-points of the edge $P_{1} P_{2}$ we do not loss in generality of the consideration.

Note, that the axis of the isometry $A$ from Lemma 1 contains $P_{1}$ and the axis of $B$ contains $P_{2}$. The aim of the construction for the polyhedron $\mathcal{P}_{n}$ is to bring its edges $P_{1} P_{2 n+2}$ and $P_{2} P_{2 n+3}$ to be axes of the respective isometries $A$ and $B$. The other vertices $P_{i}$ has to be images of $P_{1}$ and $P_{2}$ under action of $A$ and $B$. The polyhedron $\mathcal{P}_{n}$ is said to be proper if

(a) inner dihedral angles along $P_{1} P_{2 n+2}$ and $P_{2} P_{2 n+3}$ are equal to $\alpha$;

(b) the following curvilinear faces are identified by $A$ and $B$ :

$$
\begin{gathered}
A: N P_{1} P_{2} \ldots P_{2 n+2} \rightarrow N P_{1} P_{4 n+2} \ldots P_{2 n+3} P_{2 n+2}, \\
B: S P_{2} P_{1} P_{4 n+2} \ldots P_{2 n+3} \rightarrow S P_{2} P_{3} \ldots P_{2 n+3}
\end{gathered}
$$

(c) sum of the inner dihedral angles $\psi_{i}$ along $P_{i} P_{i+1}, i \in\{1, \ldots, 4 n+1\}$ equals $2 \pi$;

(d) sum of the dihedral angles $\phi_{i}$ for corresponding tetrahedra $N S P_{i} P_{i+1}$, $i \in\{1, \ldots, 4 n+1\}$ at their common edge $N S$ is $2 \pi$; 


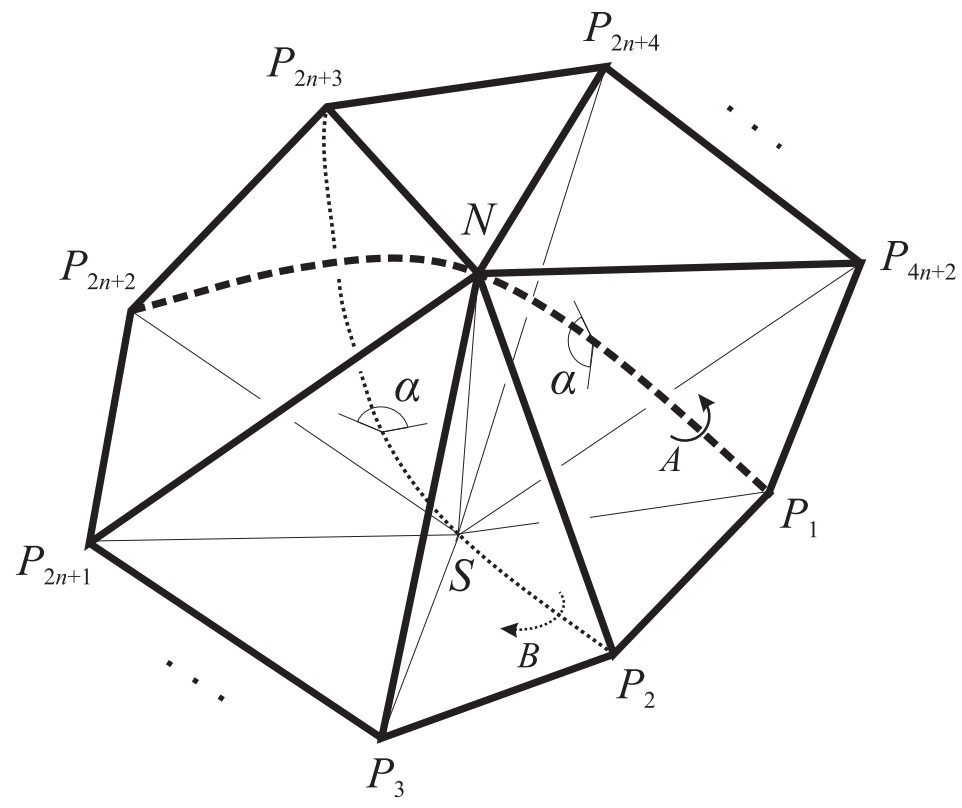

Figure 2: Fundamental polyhedron $\mathcal{P}_{n}$ for $\mathbb{T}_{n}(\alpha)$

(e) all the tetrahedra $N S P_{i} P_{i+1}$ with $i \in\{1, \ldots, 4 n+2\}, P_{4 n+3}=P_{1}$ are non-degenerated and coherently oriented.

By the orientation of a tetrahedron $N S P_{i} P_{i+1}$ one means the sign of the Gram determinant $\operatorname{det}\left(S, N, P_{i}, P_{i+1}\right)$ for corresponding quadruple $S, N, P_{i}, P_{i+1} \in$ $\mathbb{C}_{\mathbb{R}}^{2}$, where $i \in\{1, \ldots, 4 n+2\}, P_{4 n+3}=P_{1}$. A tetrahedron is non-degenerated if $\operatorname{det}\left(S, N, P_{i}, P_{i+1}\right) \neq 0$. Thus, claim (e) is satisfied if all the Gram determinants are non-zero and of the same sign.

If $\alpha=\frac{2 \pi}{m}, m \in \mathbb{N}$, then due to the Poincaré Theorem [14, Theorem 13.5.3] claims (a) - (e) imply that the group generated by the isometries $A$ and $B$ is discreet and its presentation is

$$
\Gamma=\left\langle A, B \mid(A B)^{n} A=B(A B)^{n}, A^{m}=B^{m}=\mathrm{id}\right\rangle .
$$

The metric space $\mathbb{S}_{\lambda}^{3} / \Gamma \cong \mathbb{T}_{n}\left(\frac{2 \pi}{m}\right)$ is a spherical orbifold, and $\mathcal{P}_{n}$ is its fundamental polyhedron. If $m \notin \mathbb{N}$ then the group generated by $A$ and $B$ might be non-discreet. However, the identification for the faces of $\mathcal{P}_{n}$ is of the same fashion as if it were $m \in \mathbb{N}$ and as the result one obtains the cone-manifold $\mathbb{T}_{n}(\alpha)$. By means of Lemma 1 and construction of $\mathcal{P}_{n}$ claims (a) and (b) are satisfied. For the holonomy mapping $h$ to exist the following relation should be satisfied:

$$
h\left((a b)^{n} a\right)-h\left(b(a b)^{n}\right)=(A B)^{n} A-B(A B)^{n}=0 .
$$

By Lemma 2, the condition above is satisfied if and only if

$$
U_{2 n}(\Lambda)=0,
$$


where $\Lambda=\lambda \sin \frac{\alpha}{2}$.

Thus, the parameter $\lambda$ of the metric $\mathrm{d} s_{\lambda}^{2}$ is determined completely by a root of the polynomial $U_{2 n}(\Lambda)$. From the above formula, $\lambda$ is related to the cone angle $\alpha$ by means of the equality

$$
\lambda=\frac{\Lambda}{\sin \frac{\alpha}{2}} .
$$

The roots of $U_{2 n}(\Lambda)$ are given by the following formula:

$$
\Lambda_{k}=\cos \frac{k \pi}{2 n+1},
$$

with $k \in\{1, \ldots, 2 n\}$.

The parameter $\lambda$ for the metric $\mathrm{d} s_{\lambda}^{2}$ has to be chosen in order the polyhedron $\mathcal{P}_{n}$ be proper and the metric itself be spherical.

Note, that the edges $P_{i} P_{i+1}, i \in\{1, \ldots, 4 n+2\}, P_{4 n+3}=P_{1}$ are equivalent under action of the group $\Gamma=\langle A, B\rangle$. Thus, the relation $(A B)^{n} A=B(A B)^{n}$ implies the equality

$$
\sum_{i=1}^{2(2 n+1)} \psi_{i}=2 k \pi
$$

where $k$ is an integer.

Show that one can choose $\lambda$ for the equality $k=1$ to hold for all $\alpha$ in the affirmation of the Theorem. Due to the paper [15, every two-bridge knot conemanifold with cone angle $\pi$ is a spherical orbifold. In this case all the vertices $P_{i}$ of the fundamental polyhedron belong to the same circle and all the dihedral angles $\psi_{i}$ and $\phi_{i}$ are equal to each other [12]:

$$
\phi_{i}=\psi_{i}=\frac{\pi}{2 n+1} .
$$

As far as $\cos d_{\lambda}(N, S)=\cos d_{\lambda}\left(P_{i}, P_{i+1}\right)=\lambda$, then in case $\alpha=\pi$ one obtains

$$
\lambda=\frac{\Lambda_{k}}{\sin \frac{\pi}{2}}=\cos \theta
$$

for certain $k \in\{1, \ldots, 2 n\}$ and then

$$
\sum_{i=1}^{2(2 n+1)} \psi_{i}=2(2 n+1) \theta
$$

Using the formula for the roots of $U_{2 n}(\Lambda)$ obtain that

$$
\sum_{i=1}^{2(2 n+1)} \psi_{i}=2 k \pi
$$

if $\alpha=\pi$. Thus, claim (c) for the polyhedron $\mathcal{P}_{n}$ with $\alpha=\pi$ is satisfied if $k=1$. As far as the parameter $\alpha$ varies continuously and sum of the angles $\psi_{i}$ 
represents a multiple of $2 \pi$, one has that

$$
\sum_{i=1}^{2(2 n+1)} \psi_{i}=2 \pi
$$

for all $\alpha$.

By analogy, show that with

$$
\lambda=\frac{\Lambda_{1}}{\sin \frac{\alpha}{2}}
$$

the equality

$$
\sum_{i=1}^{2(2 n+1)} \phi_{i}=2 \pi
$$

holds, that means claim (d) is also satisfied.

Verify that under conditions of the Theorem the metric $\mathrm{d} s_{\lambda}^{2}$ is spherical. This claim is equivalent to the inequality

$$
-1<\lambda<+1
$$

Note, that for

$$
\frac{2 n-1}{2 n+1} \pi<\alpha<2 \pi-\frac{2 n-1}{2 n+1} \pi
$$

it follows

$$
\sin \frac{\alpha}{2}>\sin \frac{(2 n-1) \pi}{2(2 n+1)} .
$$

As far as $\sin \frac{\alpha}{2}>0$ and $\Lambda_{1}=\sin \frac{(2 n-1) \pi}{2(2 n+1)}>0$, one has

$$
0<\lambda<1
$$

By analogy with Lemma 1 verify that

$$
C=\left(\begin{array}{ll}
0 & 1 \\
1 & 0
\end{array}\right)
$$

is an isometry of $\mathrm{d} s_{\lambda}^{2}$.

Fixed point sets of $A$ and $B$ in $\mathbb{S}_{\lambda}^{3}$ are circles

$$
\operatorname{Fix} A=\left\{\left(z_{1}, 0\right): z_{1} \in \mathbb{C},\left|z_{1}\right|=1\right\}
$$

and

$$
\text { Fix } B=\left\{\left(0, z_{2}\right): z_{2} \in \mathbb{C},\left|z_{2}\right|=1\right\},
$$

correspondingly. The geometric meaning of $C$ is that it maps the first fixed circle to the other. Thus, the relation $B=C A C^{-1}$ holds.

The following equalities

$$
P_{2 k+1}=P_{1}(A B)^{k}, k \in\{0, \ldots, n\},
$$




$$
P_{2 k}=P_{2}(A B)^{k-1}, k \in\{1, \ldots, n+1\}
$$

and

$$
\begin{gathered}
P_{2 k+1}=P_{1}(B A)^{2 n-k+1}, k \in\{n+1, \ldots, 2 n\}, \\
P_{2 k}=P_{2}(B A)^{2 n-k+2}, k \in\{n+2, \ldots, 2 n+1\},
\end{gathered}
$$

follow from the identification scheme of the edges of $\mathcal{P}_{n}$.

Define the auxiliary function

$$
\varepsilon(m)=\frac{m}{2} \alpha-\frac{4 n-m}{2} \pi .
$$

By analogy with the proof of Lemma 2 it follows that

$$
\begin{gathered}
(A B)^{k}=C(B A)^{k} C^{-1}= \\
=\left(\begin{array}{cc}
-\frac{\sin (2 k-1) \theta}{\sin \theta} e^{i \varepsilon(2 k)} & -\frac{\sin 2 k \theta}{\sin \theta} e^{i \varepsilon(2 k-1)} \\
\frac{\sin 2 k \theta}{\sin \theta} e^{i \varepsilon(2 k+1)} & \frac{\sin (2 k+1) \theta}{\sin \theta} e^{i \varepsilon(2 k)}
\end{array}\right),
\end{gathered}
$$

where $\theta=\frac{\pi}{2 n+1}$.

Suppose $N$ and $S$ to be middle-points of the edges $P_{1} P_{2 n+2}$ and $P_{2} P_{2 n+3}$, respectively. Then

$$
N=\left(e^{i \frac{\varepsilon(2 n+1)}{2}}, 0\right), S=\left(0, e^{i \frac{\varepsilon(2 n+1)}{2}}\right) .
$$

For the lengths $l_{\alpha}$ of the singular geodesic one has

$$
\cos \frac{l_{\alpha}}{4}=\left\langle P_{1}, N\right\rangle=\left\langle P_{1} C, N C\right\rangle=\left\langle P_{2}, S\right\rangle .
$$

Thus

$$
\cos \frac{l_{\alpha}}{4}=\cos \frac{(2 n+1) \alpha-(2 n-1) \pi}{4} .
$$

By construction of the polyhedron $\mathcal{P}_{n}$, the inequality $0<l_{\alpha}<4 \pi$ holds. Then it follows

$$
l_{\alpha}=(2 n+1) \alpha-(2 n-1) \pi .
$$

Given the coordinates of the vertices $P_{i}$ and the poles $N$ and $S$ of the polyhedron $\mathcal{P}_{n}$, verify claim (e).

For every four points $A, B, C, D \in \mathbb{C}_{\mathbb{R}}^{2}$, where

$$
A=\left(A_{1}, A_{2}\right), B=\left(B_{1}, B_{2}\right), C=\left(C_{1}, C_{2}\right), D=\left(D_{1}, D_{2}\right),
$$

their Gram determinant is

$$
\operatorname{det}(A, B, C, D):=\operatorname{det}\left(\begin{array}{cccc}
\operatorname{Re} A_{1} & \operatorname{Im} A_{1} & \operatorname{Re} A_{2} & \operatorname{Im} A_{2} \\
\operatorname{Re} B_{1} & \operatorname{Im} B_{1} & \operatorname{Re} B_{2} & \operatorname{Im} B_{2} \\
\operatorname{Re} C_{1} & \operatorname{Im} C_{1} & \operatorname{Re} C_{2} & \operatorname{Im} C_{2} \\
\operatorname{Re} D_{1} & \operatorname{Im} D_{1} & \operatorname{Re} D_{2} & \operatorname{Im} D_{2}
\end{array}\right)
$$


Each tetrahedron $N S P_{i} P_{i+1}$ with $i \in\{1, \ldots, 2 n+1\}$ is isometric to $N S P_{2 n+i+1} P_{2 n+i+2}, i \in\{1, \ldots, 2 n+1\}, P_{4 n+3}=P_{1}$ by means of the isometry $C$ defined above. Thus, we consider only the tetrahedra $N S P_{i} P_{i+1}$ with $i \in\{1, \ldots, 2 n+1\}$. Split them into two groups: the tetrahedra $N S P_{2 k+1} P_{2 k+2}$ with $k \in\{0, \ldots, n\}$ and the tetrahedra $N S P_{2 k} P_{2 k+1}$ with $k \in\{1, \ldots, n\}$.

Substitute $\alpha=\beta+\pi$ and proceed with straightforward calculations:

$$
\begin{aligned}
\Delta_{k}^{(1)}(\beta)=\operatorname{det}(S, & \left.N, P_{2 k+1}, P_{2 k+2}\right)=\cos ^{2} \frac{L_{1} \beta}{4}-U_{2 k-1}^{2}(\cos \theta) \sin ^{2} \frac{\beta}{2}= \\
= & T_{L_{1}}^{2}\left(\cos \frac{\beta}{4}\right)-U_{2 k-1}^{2}(\cos \theta) \sin ^{2} \frac{\beta}{2},
\end{aligned}
$$

where $k \in\{0, \ldots, n\}, L_{1}=|2 n-4 k+1|, \theta=\frac{\pi}{2 n+1}, \beta \in[-2 \theta, 2 \theta]$;

$$
\begin{aligned}
\Delta_{k}^{(2)}(\beta)=\operatorname{det}(S & \left., N, P_{2 k}, P_{2 k+1}\right)=\cos ^{2} \frac{L_{2} \beta}{4}-U_{2 k-2}^{2}(\cos \theta) \sin ^{2} \frac{\beta}{2}= \\
= & T_{L_{2}}^{2}\left(\cos \frac{\beta}{4}\right)-U_{2 k-1}^{2}(\cos \theta) \sin ^{2} \frac{\beta}{2},
\end{aligned}
$$

where $k \in\{1, \ldots, n\}, L_{2}=|2 n-4 k+3|, \theta$ and $\beta$ the same as above. The first kind Chebyshev polynomial of degree $k \geq 0$ is denoted by $T_{k}$. Assume that

$$
U_{-1}(\cos \theta)=0, U_{0}(\cos \theta)=1
$$

for the sake of brevity.

All the functions $\Delta_{k}^{(j)}(\beta), j \in\{1,2\}$ are even on the interval $[-2 \theta, 2 \theta]$. Then one considers them only on the interval $[0,2 \theta]$. Note, that the polynomial $T_{L_{j}}^{2}(\cos \beta)$ monotonously decreases and the function $\sin ^{2} \frac{\beta}{2}$ monotonously increases with $\beta \in[0,2 \theta]$. Moreover, $T_{L_{j}}^{2}(\cos 0)=T_{L_{j}}^{2}(1)=1$. Then it follows that $\Delta_{k}^{(j)}(\beta)>0$ with $\beta \in(-2 \theta, 2 \theta)$. Also, one has $\Delta_{k}^{(j)}( \pm 2 \theta)=0$.

Then for all $\beta \in(-2 \theta, 2 \theta)$ (i.e. for all $\alpha$ in the affirmation of the Theorem)

$$
\operatorname{det}\left(S, N, P_{i}, P_{i+1}\right)>0
$$

where $i \in\{1, \ldots, 4 n+2\}, P_{4 n+3}=P_{1}$. Thus, claim (e) for the polyhedron $\mathcal{P}_{n}$ is satisfied.

Use the Schläfli formula [16] to obtain the volume formula for $\mathbb{T}_{n}(\alpha)$. One has

$$
\mathrm{d} \mathbb{V o l} \mathbb{T}_{n}(\alpha)=\frac{l_{\alpha}}{2} \mathrm{~d} \alpha=\frac{(2 n+1) \alpha-(2 n-1) \pi}{2} \mathrm{~d} \alpha .
$$

Note, that $\operatorname{Vol}_{n}(\alpha) \rightarrow 0$ with $\alpha \rightarrow \frac{2 n-1}{2 n+1} \pi$. In this case $d_{\lambda}\left(P_{i}, P_{i+1}\right) \rightarrow 0$, where $i \in\{1, \ldots, 4 n+2\}, P_{4 n+3}=P_{1}$ and the fundamental polyhedron collapses to a point. Thus

$$
\operatorname{Vol} \mathbb{T}_{n}(\alpha)=\frac{1}{2 n+1}\left(\frac{2 n+1}{2} \alpha-\frac{2 n-1}{2} \pi\right)^{2} .
$$

Remark 1 The domain of the spherical metric existence in Theorem 11 was indicated before in [10, Proposition 2.1]. 


\section{Torus links $\mathbb{L}_{n}$}

Let $\mathbb{L}_{n}, n \geq 2$ be a torus link $\mathrm{t}(2 n, 2)$ with two components. The corresponding link in the rational census is $2 n / 1$ (Fig. 3). The fundamental group of $\mathbb{L}_{n}$ is presented as

$$
\pi_{1}\left(\mathbb{S}^{3} \backslash \mathbb{L}_{n}\right)=\left\langle a, b \mid(a b)^{n}=(b a)^{n}\right\rangle .
$$

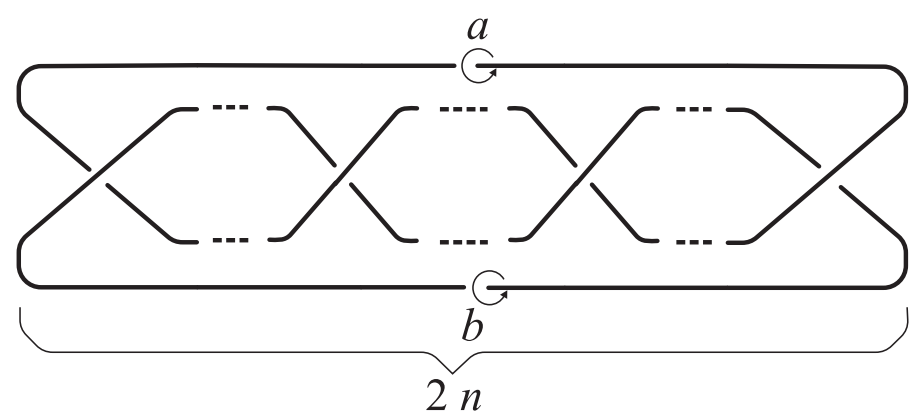

Figure 3: Link 2n/1

Let $\mathbb{L}_{n}(\alpha, \beta)$ denote a cone-manifold with singular locus the link $\mathbb{L}_{n}$ and the cone angles $\alpha, \beta$ along its components.

For every $\alpha, \beta \in(0,2 \pi)$ and $\lambda \in(-1,+1)$, we denote

$$
A=\left(\begin{array}{cc}
1 & 0 \\
-2 i e^{i \frac{\alpha}{2}} \lambda \sin \frac{\alpha}{2} & e^{i \alpha}
\end{array}\right)
$$

and

$$
B=\left(\begin{array}{cc}
e^{i \beta} & -2 i e^{i \frac{\beta}{2}} \lambda \sin \frac{\beta}{2} \\
0 & 1
\end{array}\right) .
$$

By Lemma 1, linear transformations $A$ and $B$ are isometries of $\mathbb{S}_{\lambda}^{3}$.

Lemma 3 For every integer $n \geq 2$ the following equality holds

$$
(A B)^{n}-(B A)^{n}=4 U_{n-1}(\Lambda) \lambda e^{i\left(\frac{\alpha+\beta}{2}+\pi\right) n} \sin \frac{\alpha}{2} \sin \frac{\beta}{2} M,
$$

where $M$ is a non-zero $2 \times 2$ matrix and $U_{n-1}(\Lambda)$ is the second kind Chebyshev polynomial of degree $n-1$ in variable

$$
\Lambda=\left(1-\lambda^{2}\right) \cos \frac{\alpha-\beta}{2}+\lambda^{2} \cos \frac{\alpha+\beta}{2} .
$$

Proof. By analogy with Lemma 2 ,

With Lemma 3 the main theorem of the section follows: 
Theorem 2 The cone-manifold $\mathbb{L}_{n}(\alpha, \beta), n \geq 2$ is spherical if

$$
\begin{aligned}
& -2 \pi\left(1-\frac{1}{n}\right)<\alpha-\beta<2 \pi\left(1-\frac{1}{n}\right), \\
& 2 \pi\left(1-\frac{1}{n}\right)<\alpha+\beta<2 \pi\left(1+\frac{1}{n}\right) .
\end{aligned}
$$

The lengths $l_{\alpha}, l_{\beta}$ of its singular geodesics (i.e. lengths of the components for $\mathbb{L}_{n}$ ) are equal to each other and

$$
l_{\alpha}=l_{\beta}=\frac{\alpha+\beta}{2} n-\pi(n-1) .
$$

The volume of $\mathbb{L}_{n}(\alpha, \beta)$ is

$$
\mathbb{V o l}_{n}(\alpha, \beta)=\frac{1}{2 n}\left(\frac{\alpha+\beta}{2} n-(n-1) \pi\right)^{2} .
$$

Proof. One continues the proof by analogy with Theorem 1 .

Suppose that $\mathbb{L}_{n}(\alpha, \beta)$ is spherical. Then there exists a holonomy mapping [1]:

$$
\begin{gathered}
h: \pi_{1}\left(\mathbb{S}^{3} \backslash \mathbb{L}_{n}\right) \longmapsto \operatorname{Isom} \mathbb{S}_{\lambda}^{3}, \\
h(a)=A, h(b)=B .
\end{gathered}
$$

Also,

$$
h\left((a b)^{n}\right)-h\left((b a)^{n}\right)=(A B)^{n}-(B A)^{n}=0 .
$$

By means of Lemma 3 the equality above holds either if $\lambda=0$, or if

$$
\Lambda=\left(1-\lambda^{2}\right) \cos \frac{\alpha-\beta}{2}+\lambda^{2} \cos \frac{\alpha+\beta}{2}
$$

is a root of the equation $U_{n-1}(\Lambda)=0$.

In case $\lambda=0$ the image of $h$ is abelian, because of the additional relation $A B=$ $B A$. With $n \geq 2$ this leads to a degenerate geometric structure. Thus, one has to choose the parameter $\lambda$ for the metric $\mathrm{d} s_{\lambda}^{2}$ using roots of the Chebyshev polynomial $U_{n-1}(\Lambda)$.

The fundamental polyhedron $\mathcal{F}_{n}$ for the cone-manifold $\mathbb{L}_{n}(\alpha, \beta)$ is depicted at Fig. 4. Suppose its vertices $P_{1}$ and $P_{2}$ to be

$$
P_{1}=(1,0), P_{2}=(0,1) .
$$

The axes of isometries $A$ and $B$ correspond to the edges $P_{1} P_{2 n+1}$ and $P_{2} P_{2 n+2}$. Points $N$ and $S$ are respective middles of the edges $P_{1} P_{2 n+1}$ and $P_{2} P_{2 n+2}$. Those are called North and South poles of the polyhedron.

The polyhedron $\mathcal{F}_{n}$ is said to be proper if

(a) respective inner dihedral angles along the edges $P_{1} P_{2 n+1}$ and $P_{2} P_{2 n+2}$ are equal to $\alpha$ and $\beta$; 


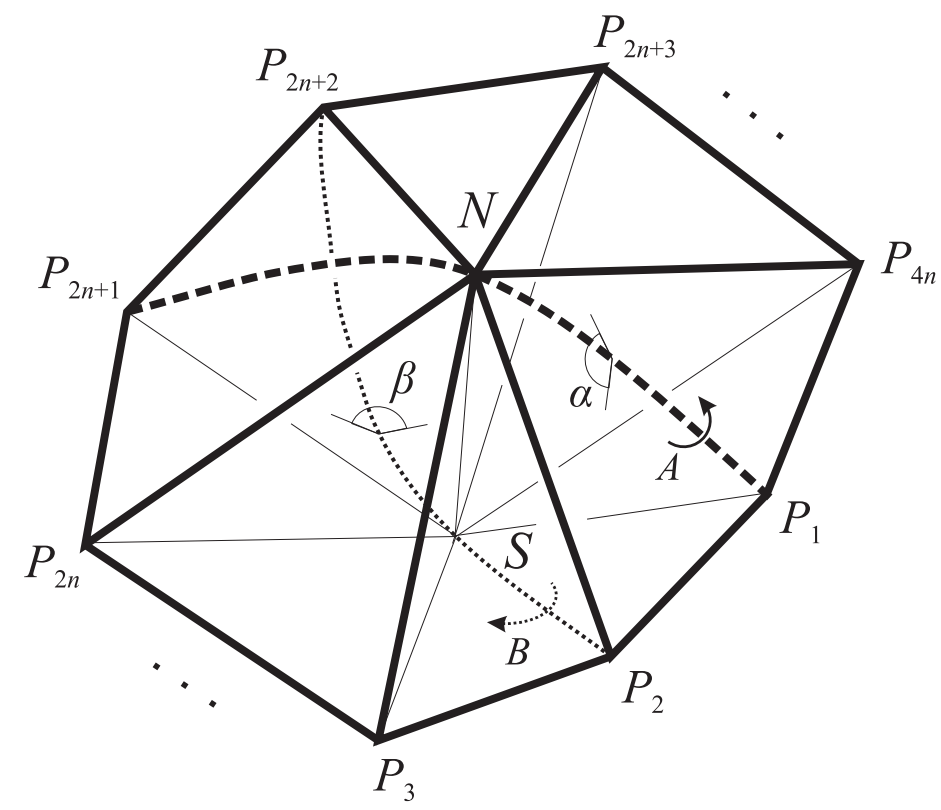

Figure 4: The fundamental polyhedron $\mathcal{F}_{n}$ for $\mathbb{L}_{n}(\alpha, \beta)$

(b) curvilinear faces of the polyhedron are identified by $A$ and $B$ :

$$
\begin{gathered}
A: N P_{1} P_{2} \ldots P_{2 n+1} \rightarrow N P_{1} P_{4 n} \ldots P_{2 n+2} P_{2 n+1}, \\
B: S P_{2} P_{1} P_{4 n} \ldots P_{2 n+2} \rightarrow S P_{2} P_{3} \ldots P_{2 n+2} ;
\end{gathered}
$$

(c) sum of the inner dihedral angles $\psi_{i}$ along the edges $P_{i} P_{i+1}$, $i \in\{1, \ldots, 4 n-1\}$ equals $2 \pi$;

(d) sum of the dihedral angles $\phi_{i}$ for tetrahedra $N S P_{i} P_{i+1}, i \in\{1, \ldots, 4 n-$ $1\}$ at their common edge $N S$ equals $2 \pi$;

(e) all the tetrahedra $N S P_{i} P_{i+1}$ with $i \in\{1, \ldots, 4 n\}, P_{4 n+1}=P_{1}$ are non-degenerated and coherently oriented.

In order to choose the parameter $\lambda$ for the corresponding metric consider the fundamental polyhedron $\mathcal{F}_{n}$ with $\alpha=\beta=\pi$. Then all its vertices belong to the same circle and all the dihedral angles $\psi_{i}$ of the tetrahedra $N S P_{i} P_{i+1}$ along the edges $P_{i} P_{i+1}$ are equal to $\psi=\frac{\pi}{2 n}$ [12. Also the dihedral angles $\phi_{i}$ of the tetrahedra $N S P_{i} P_{i+1}$ along their common edge $N S$ are equal to each other:

$$
\phi_{i}=\phi=\frac{\pi}{2 n} \text {. }
$$

In this case $\lambda=\left\langle P_{1}, P_{2}\right\rangle=\cos \phi$ and

$$
\Lambda=-\cos 2 \phi=\cos \frac{(n-1) \pi}{n} .
$$


All the roots of $U_{n-1}(\Lambda)$ are given by the formula

$$
\Lambda_{k}=\cos \frac{k \pi}{n}, k \in\{1, \ldots, n-1\},
$$

so one choose the root $\Lambda_{k}$ with $k=n-1$. Then, by analogy with Theorem 1 equalities

$$
\sum_{i=1}^{4 n} \psi_{i}=2 \pi
$$

and

$$
\sum_{i=1}^{4 n} \phi_{i}=2 \pi
$$

are satisfied at the point $\alpha=\beta=\pi$ of the domain

$$
\mathcal{D}=\left\{(\alpha, \beta):|\alpha-\beta|<2 \pi\left(1-\frac{1}{n}\right),|\alpha+\beta-2 \pi|<\frac{2 \pi}{n}\right\},
$$

depicted at Fig. 5 .

In terms of the parameter $\lambda$, that defines the metric $\mathrm{d} s_{\lambda}^{2}$, one has

$$
\lambda^{2}=\frac{\cos \frac{\alpha-\beta}{2}+\cos \frac{\pi}{n}}{\cos \frac{\alpha-\beta}{2}-\cos \frac{\alpha+\beta}{2}} .
$$

As for all $(\alpha, \beta) \in \mathcal{D}$ the inequality $0<\lambda^{2}<1$ is satisfied, the metric $\mathrm{d} s_{\lambda}^{2}$ is spherical regarding the corresponding domain. By analogy with Theorem 1 one can show that claims (a) - (d) for the polyhedron $\mathcal{F}_{n}$ are satisfied in the interior of $\mathcal{D}$.

The lengths $l_{\alpha}$ and $l_{\beta}$ of singular geodesics for the cone-manifold $\mathbb{L}_{n}(\alpha, \beta)$ meet the relations

$$
\begin{aligned}
& \cos \frac{l_{\alpha}}{2}=\left\langle P_{1}, N\right\rangle, \\
& \cos \frac{l_{\beta}}{2}=\left\langle P_{2}, S\right\rangle .
\end{aligned}
$$

By analogy with the proof of Theorem 1 one obtains

$$
l_{\alpha}=l_{\beta}=\frac{\alpha+\beta}{2} n-\pi(n-1) .
$$

Given the coordinates of the vertices for the fundamental polyhedron verify claim (e) for all $(\alpha, \beta)$ in the domain $\mathcal{D}$.

Make use of the Schläfli formula [16] to obtain the volume of $\mathbb{L}_{n}(\alpha, \beta)$ :

$$
\mathrm{d} \mathbb{V}_{\mathrm{ol}} \mathbb{L}_{n}(\alpha, \beta)=\frac{l_{\alpha}}{2} \mathrm{~d} \alpha+\frac{l_{\beta}}{2} \mathrm{~d} \beta=\left(\frac{\alpha+\beta}{2} n-\pi(n-1)\right) \mathrm{d}\left(\frac{\alpha+\beta}{2}\right) .
$$

Note, that with

$$
\alpha=\beta \rightarrow \pi \frac{n-1}{n}
$$

the fundamental polyhedron $\mathcal{F}_{n}$ collapses to a point (i.e. the volume tends to 0 ). The last affirmation of the Theorem follows. 


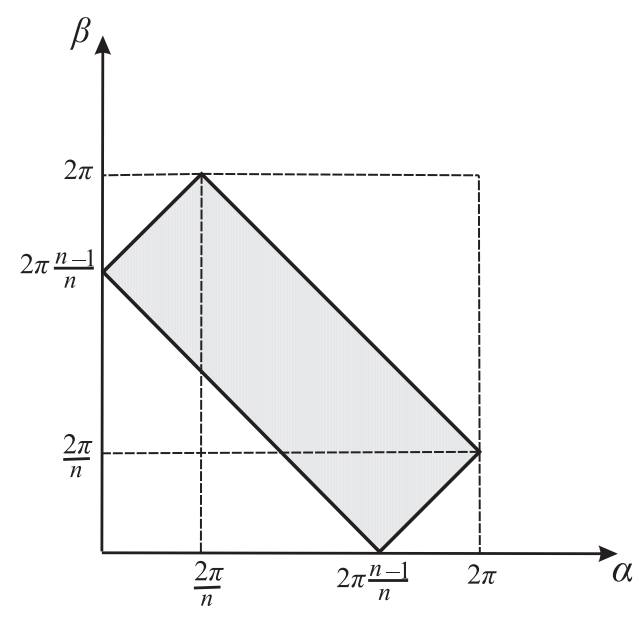

Figure 5: The domain $\mathcal{D}$ of sphericity for $\mathbb{L}_{n}(\alpha, \beta)$

Remark 2 Under condition $\alpha=\beta$ the inequality from the affirmation of Theorem 圆 coincides with the inequality from [10, Proposition 2.2].

Remark 3 Note, that the lengths of the singular geodesics for $\mathbb{L}_{n}(\alpha, \beta)$ are equal even if $\alpha \neq \beta$.

\section{References}

[1] Thurston W. P. The geometry and topology of 3-manifolds. Princeton: Lecture Notes, 1977-78. Available on-line.

[2] Rolfsen D. Knots and links. Berkeley: Publish or Perish Inc., 1976. Google Books.

[3] Thurston W. P. Hyperbolic geometry and 3-manifolds. Cambridge: Cambridge Univ. Press, 1982. (London Math. Soc. Lect. Note Ser.; 48, 9-25).

[4] Neumann W. P. Notes on geometry and 3-manifolds, with appendix by Paul Norbury, in: Low Dimensional Topology, Böröczky, Neumann, Stipsicz, Eds. // Bolyai Society Mathematical Studies. 1999. V. 8. P. 191-267. Available on-line.

[5] Seifert H., Weber C. Die beiden Dodecaederräme // Math. Z. 1933. V. 37. P. 237-253. Available on-line.

[6] Dunbar W. D. Geometric orbifolds // Rev. Mat. Univ. Complut. Madrid. 1988. V. 1. P. 67-99. Available on-line. 
[7] Derevnin D., Mednykh A., Mulazzani M. Geometry of trefoil conemanifold // Preprint. 2007.

[8] Hilden H. M., Lozano M. T., Montesinos-Amilibia J. M. On a remarkable polyhedron geometrizing the figure eight cone manifolds // J. Math. Sci. Univ. Tokyo. 1995. V. 2. P. 501-561. Available on-line.

[9] Mednykh A., Rasskazov A. Volumes and degeneration of cone-structures on the figure-eight knot // Tokyo J. of Math. 2006. V. 29. N. 2. P. 445464.

[10] Porti J. Spherical cone structures on 2-bridge knots and links // Kobe J. of Math. 2004. V. 21. N. 1. P. 61-70. Available on-line.

[11] Molnár E. The projective interpretation of the eight 3-dimensional homogeneous geometries // Beiträge zur Algebra und Geometrie. 1997. V. 38. N. 2. P. 261-288. Available on-line.

[12] Mednykh A., Rasskazov A. On the structure of the canonical fundamental set for the 2-bridge link orbifolds // Preprint. 1996. Available on-line.

[13] Minkus J. The branched cyclic coverings of 2-bridge knots and links // Mem. Amer. Math. Soc. 1982. V. 35. N. 255. Google Books.

[14] Ratcliffe J. Foundations of hyperbolic manifolds. New York: SpringerVerlag, 1994. (Graduate Texts in Math.; 149). Google Books.

[15] Hodgson C., Rubinstein J. H. Involutions and isotopies of lens spaces, Knot theory and manifolds (Vancouver, B.C., 1983). Berlin: SpringerVerlag, 1985. (Lecture Notes in Math.; 1144, 60-96).

[16] Hodgson C. Degeneration and regeneration of hyperbolic structures on three-manifolds // Princeton: Thesis, 1986.

Alexander Kolpakov

Novosibirsk State University

630090, Pirogova str., bld. 2

Novosibirsk, Russia

kolpakov.alexander@gmail.com

Alexander Mednykh

Sobolev Institute of Mathematics, SB RAS

630090, Koptyug avenue, bld. 4,

Novosibirsk, Russia

mednykh@math.nsc.ru 